\title{
Early dose response analysis of ocular hypotensive effects of propranolol in patients with ocular hypertension
}

\author{
KARIN WETTRELL AND MAURIZIO PANDOLFI \\ From the Department of Ophthalmology, Hospital of Malmö, \\ University of Lund, Sweden
}

Cumulative evidence indicates that propranolol

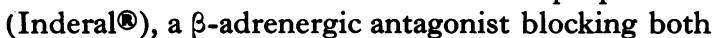
$\beta_{1}$ and $\beta_{2}$ receptors, decreases the intraocular pressure (IOP). This substance has been administered topically (Musini, Fabbri, Bergamaschi, Mandelli, and Shanks, 1971; Bietti, 1972; Vale, Gibbs, and Phillips, I972), intravenously (Phillips, Howitt, and Rowlands, 1967; Vale and Phillips, 1970; Sharaf, Haroun, Ishaac, Shewy, and Nassef, 1974), and orally (Phillips and others, 1967; Coté and Drance, 1968; Öhrström, 1973; Pandolfi and Öhrström, 1974; Wettrell and Pandolfi, 1975), both in healthy volunteers and in patients with ocular hypertension or glaucoma. No information is available on the dose-intraocular pressure response to administration of propranolol.

The aim of the present study was to determine the time-course and dose-response of the intraocular pressure of propranolol administered orally to patients with ocular hypertension.

\section{Materials and methods}

The trial was performed in a single-blind manner with the patients being unaware of the dose level employed. Two groups each comprising six patients with ocular hypertension were studied. The first group consisted of four women and two men aged between 65 and 69 years with an IOP ranging from 20 to $29 \mathrm{mmHg}$ (Group A). The second group consisted of five women and one man aged between 43 and 69 years with an IOP ranging from 30 to $39 \mathrm{mmHg}$ (Group B). At 8 a.m. on the first day of the trial two people in Group A were found to have one single IOP value exceeding that for the group (one had an IOP of $30 \mathrm{mmHg}$, the other $32 \mathrm{mmHg}$ ) but as in neither case was this later observed to rise above $30 \mathrm{mmHg}$, these persons were included in Group A. All previous treatment, ocular or otherwise, was stopped at least 24 hours before the beginning of the trial. On the initial visit the patients were questioned on previous history of asthma, brochospasm, or other states contraindicating adrenergic beta-blockers. Cardiovascular examination was performed and electrocardiograms taken to ascertain their suitability for the study.

Propranolol or placebo was administered orally in a

Address for reprints: Karin Wettrell, MD, Department of Ophthalmology, Hospital of Malmö, University of Lund, 214 or Malmö, Sweden single dose at 9 a.m. after fasting 12 hours. Two hours later, a light lunch was served. Placebo and propranolol in doses of 20,40 , and $80 \mathrm{mg}$ were tested at intervals of at least 48 hours.

One hour before and each hour after administration of propranolol or placebo the IOP was tested using a Goldmann applanation tonometer. After some rest in supine position the systemic blood pressure and pulse rate were recorded hourly. The tonometer was calibrated daily and the systemic blood pressure was measured with a sphygomanometer which had previously been tested at the local department of medicine.

All results were expressed as the arithmetic mean \pm the standard error of the mean. The method of least squares (least square line) was used to obtain the doseresponse curve. The significance of differences was calculated by Student's $t$ test.

\section{Results}

In both groups a decrease in mean IOP was noted after one hour, reaching its maximum after three hours. The decrease in IOP showed a clear dosecorrelation (Tables I and II; Figs I and 2). The maximum mean IOP reduction after 20,40 , and $80 \mathrm{mg}$ propranolol in Group A was $6 \cdot 2,6 \cdot 9$, and $8.5 \mathrm{mmHg}$, respectively. In Group $B$ the corresponding decrease was $5.8,7.8$, and $9.8 \mathrm{mmHg}$. A similar decrease in mean IOP was also found in the fellow eye. Six hours after administration of propranolol the mean IOP in both groups was still reduced, the reduction still being proportional to the dose administered.

The mean systolic blood pressure decrease was only moderate (Table III). The maximum fall was observed between three and four hours after administration of 20,40 , and $80 \mathrm{mg}$ propranolol; in Group $A$ it was 10,25 , and $15 \mathrm{mmHg}$, and in Group B 13, 15, and $16 \mathrm{mmHg}$, respectively. The reduction showed no obvious dose-relationship and was never statistically significant with one exception (40 $\mathrm{mg}$ propranolol three hours after administration). The absolute fall in mean diastolic pressure was even slighter and did not exceed Io $\mathrm{mmHg}$ for any dose level studied. Statistical significance was never attained.

The decrease in pulse rate was appreciable one hour after administration of propranolol and 
Table I The time-course of the IOP response of six ocular hypertensive patients (20 to $29 \mathrm{mmHg}$ ) after treatment with placebo and different doses of propranolol

\begin{tabular}{|c|c|c|c|c|c|c|c|}
\hline \multicolumn{8}{|c|}{$\begin{array}{c}\text { Initial highest IOP } \\
\text { Hours }\end{array}$} \\
\hline Dosage & $-I$ & $\circ$ & I & 2 & 3 & 4 & 6 \\
\hline Placebo & $\begin{array}{l}27 \cdot 3 \\
\pm 1 \cdot 26\end{array}$ & $\begin{array}{l}25 \cdot 5 \\
\pm 1 \cdot 02\end{array}$ & $\begin{array}{l}24 \cdot 2 \\
\pm 0 \cdot 95\end{array}$ & $\begin{array}{l}23 \cdot 5 \\
\pm 1 \cdot 26\end{array}$ & $\begin{array}{l}23 \cdot 2 \\
\pm r \cdot 56\end{array}$ & $\begin{array}{l}24 \cdot 5 \\
\pm 1 \cdot 09\end{array}$ & $\begin{array}{l}24 \cdot 3 \\
\pm 0.61\end{array}$ \\
\hline $20 \mathrm{mg}$ & $\begin{array}{c}24 \cdot 7 \\
\pm 1 \cdot 15\end{array}$ & $\begin{array}{c}24.3 \\
\pm 0.76\end{array}$ & $\begin{array}{l}20.8^{*} \\
\pm 0.60\end{array}$ & $\begin{array}{r}18.3 \dagger \\
\pm 0.42\end{array}$ & $\begin{array}{r}17 \cdot 0 \dagger \\
\pm 1 \cdot 00\end{array}$ & $\begin{array}{r}19.3 \dagger \\
\pm 0.56\end{array}$ & $\begin{array}{l}22 \cdot 0 \\
\pm 1 \cdot 53\end{array}$ \\
\hline $40 \mathrm{mg}$ & $\begin{array}{c}25 \cdot 7 \\
\pm x \cdot 09\end{array}$ & $\begin{array}{c}24.5 \\
\pm 0.62\end{array}$ & $\begin{array}{c}20 \cdot 2^{*} \\
\pm 1 \cdot 19\end{array}$ & $\begin{array}{r}17.0+ \\
\pm 0.68\end{array}$ & $\begin{array}{r}16.3 \dagger \\
\pm 0.80\end{array}$ & $\begin{array}{r}17.5 \ddagger \\
\pm 0.50\end{array}$ & $\begin{array}{r}19.3 \dagger \\
\pm 1.02\end{array}$ \\
\hline $80 \mathrm{mg}$ & $\begin{array}{l}26 \cdot 0 \\
\pm 0 \cdot 58\end{array}$ & $\begin{array}{l}24.8 \\
\pm 0.98\end{array}$ & $\begin{array}{r}16 \cdot 7 \ddagger \\
\pm 0 \cdot 71\end{array}$ & $\begin{array}{r}15.3 \ddagger \\
\pm 0.67\end{array}$ & $\begin{array}{r}14 \cdot 7 \ddagger \\
\pm 0 \cdot 33\end{array}$ & $\begin{array}{r}15.3 \ddagger \\
\pm 0.99\end{array}$ & $\begin{array}{r}17 \cdot 0 \ddagger \\
\pm 1 \cdot 29\end{array}$ \\
\hline $\begin{array}{l}\text { Fellow eye } \\
\text { Placebo }\end{array}$ & $\begin{array}{l}23 \cdot 8 \\
\pm 1 \cdot 25\end{array}$ & $\begin{array}{l}2 \mathrm{I} \cdot 8 \\
\pm \mathrm{I} \cdot \mathrm{II}\end{array}$ & $\begin{array}{l}20 \cdot 5 \\
\pm 1 \cdot 18\end{array}$ & $\begin{array}{l}20 \cdot 7 \\
\pm 1 \cdot 20\end{array}$ & $\begin{array}{l}20 \cdot 3 \\
\pm 1 \cdot 33\end{array}$ & $\begin{array}{l}20 \cdot 8 \\
\pm 0.91\end{array}$ & $\begin{array}{l}22 \cdot 7 \\
\pm 1 \cdot 54\end{array}$ \\
\hline $20 \mathrm{mg}$ & $\begin{array}{c}21 \cdot 8 \\
\pm 0.60\end{array}$ & $\begin{array}{c}21 \cdot 5 \\
\pm 0.76\end{array}$ & $\begin{array}{c}19.2 \\
\pm 0.60\end{array}$ & $\begin{array}{r}17.5^{*} \\
\pm 0.43\end{array}$ & $\begin{array}{c}17 \cdot 0 \\
\pm 0.93\end{array}$ & $\begin{array}{r}17.3 \dagger \\
\pm 0.21\end{array}$ & $\begin{array}{c}19.5 \\
\pm 0.67\end{array}$ \\
\hline $40 \mathrm{mg}$ & $\begin{array}{l}21 \cdot 7 \\
\pm 0 \cdot 61\end{array}$ & $\begin{array}{l}21 \cdot 3 \\
\pm 0 \cdot 71\end{array}$ & $\begin{array}{l}18 \cdot 0 \\
\pm 1 \cdot 03\end{array}$ & $\begin{array}{l}16.8^{*} \\
\pm 0.65\end{array}$ & $\begin{array}{r}15 \cdot 3 \dagger \\
\pm 0.80\end{array}$ & $\begin{array}{l}16 \cdot 0 \dagger \\
\pm 0.86\end{array}$ & $\begin{array}{l}17.8^{*} \\
\pm 0.87\end{array}$ \\
\hline $80 \mathrm{mg}$ & $\begin{array}{l}21 \cdot 7 \\
\pm 0 \cdot 42\end{array}$ & $\begin{array}{l}21 \cdot 7 \\
\pm 1 \cdot 15\end{array}$ & $\begin{array}{l}15 \cdot 7^{*} \\
\pm 1 \cdot 20\end{array}$ & $\begin{array}{l}14 \cdot 0 \dagger \\
\pm 1 \cdot 03\end{array}$ & $\begin{array}{r}13.7 \dagger \\
\pm 0.92\end{array}$ & $\begin{array}{r}14.7 \ddagger \\
\pm 0.92\end{array}$ & $\begin{array}{r}15.3 \dagger \\
\pm 1.02\end{array}$ \\
\hline
\end{tabular}

Values expressed as mean $\pm \mathrm{SEM}$ ( $\mathrm{mmHg}$ ). Statistical significance expressed as * $(P<0.05) ; \dagger(P<0.01) ; \ddagger P<0.001)$

Table II The time-course of the IOP response of six ocular hypertensive patients ( 30 to $39 \mathrm{mmHg}$ ) after treatment with placebo and different doses of propranolol

\begin{tabular}{|c|c|c|c|c|c|c|c|}
\hline \multicolumn{8}{|c|}{$\begin{array}{r}\text { Initial highest IOP } \\
\text { Hours }\end{array}$} \\
\hline (1) & $-I$ & 0 & I & 2 & 3 & 4 & 6 \\
\hline Placebo & $\begin{array}{c}32 \cdot 2 \\
\pm 0 \cdot 98\end{array}$ & $\begin{array}{l}31 \cdot 3 \\
\pm 0 \cdot 71\end{array}$ & $\begin{array}{c}28 \cdot 5 \\
\pm 0.99\end{array}$ & $\begin{array}{l}29 \cdot 5 \\
\pm 1 \cdot 48\end{array}$ & $\begin{array}{l}28 \cdot 0 \\
\pm I \cdot 10\end{array}$ & $\begin{array}{l}27 \cdot 7 \\
\pm 1 \cdot 15\end{array}$ & $\begin{array}{c}30 \cdot 2 \\
\pm 1 \cdot 54\end{array}$ \\
\hline $20 \mathrm{mg}$ & $\begin{array}{l}32 \cdot 2 \\
\pm 1 \cdot 28\end{array}$ & $\begin{array}{l}3 r \cdot 8 \\
\pm I \cdot 94\end{array}$ & $\begin{array}{c}29 \cdot 3 \\
\pm I \cdot 6 r\end{array}$ & $\begin{array}{r}23 \cdot 2 \dagger \\
\pm 1 \cdot 19\end{array}$ & $\begin{array}{r}22 \cdot 2^{*} \\
\pm 1 \cdot 62\end{array}$ & $\begin{array}{c}25 \cdot 8 \\
\pm 1 \cdot 25\end{array}$ & $\begin{array}{l}26 \cdot 0^{*} \\
\pm 0 \cdot 68\end{array}$ \\
\hline $40 \mathrm{mg}$ & $\begin{array}{l}33 \cdot 5 \\
\pm 1 \cdot 18\end{array}$ & $\begin{array}{l}30 \cdot 8 \\
\pm \mathbf{I} \cdot 70\end{array}$ & $\begin{array}{l}24 \cdot 8 \\
\pm I \cdot 35\end{array}$ & $\begin{array}{r}22 \cdot 0 \dagger \\
\pm 1 \cdot 03\end{array}$ & $\begin{array}{r}20 \cdot 2 \ddagger \\
\pm 1 \cdot 05\end{array}$ & $\begin{array}{r}21 \cdot 5 \dagger \\
\pm 1 \cdot 38\end{array}$ & $\begin{array}{l}25 \cdot 0^{*} \\
\pm 1 \cdot 67\end{array}$ \\
\hline $80 \mathrm{mg}$ & $\begin{array}{r}32 \cdot 0 \\
\pm 1 \cdot 24 \\
\end{array}$ & $\begin{array}{r}32 \cdot 3 \\
\pm 1 \cdot 7 x \\
\end{array}$ & $\begin{array}{r}23 \cdot 8 * \\
\pm 1.82 \\
\end{array}$ & $\begin{array}{r}19.0 \ddagger \\
\pm 0.93 \\
\end{array}$ & $\begin{array}{r}18.2 \ddagger \\
\pm 0.79 \\
\end{array}$ & $\begin{array}{r}19 \cdot 2 \ddagger \\
\pm 0.95\end{array}$ & $\begin{array}{r}21 \cdot 0 \pm \\
\pm 0.77 \\
\end{array}$ \\
\hline $\begin{array}{l}\text { Fellow eye } \\
\text { Placebo }\end{array}$ & $\begin{array}{l}30 \cdot 7 \\
\pm 0.88\end{array}$ & $\begin{array}{l}29 \cdot 3 \\
\pm 0 \cdot 80\end{array}$ & $\begin{array}{l}26 \cdot 8 \\
\pm 0 \cdot 79\end{array}$ & $\begin{array}{l}26 \cdot 7 \\
\pm 0 \cdot 84\end{array}$ & $\begin{array}{l}26.8 \\
\pm 0.79\end{array}$ & $\begin{array}{l}26 \cdot 2 \\
\pm 1 \cdot 19\end{array}$ & $\begin{array}{l}26 \cdot 8 \\
\pm 1 \cdot 14\end{array}$ \\
\hline $20 \mathrm{mg}$ & $\begin{array}{c}30.5 \\
\pm 0.92\end{array}$ & $\begin{array}{l}30 \cdot 2 \\
\pm 0 \cdot 70\end{array}$ & $\begin{array}{l}26 \cdot 8 \\
\pm \mathrm{I} \cdot 01\end{array}$ & $\begin{array}{r}22 \cdot 7^{*} \\
\pm \mathbf{I} \cdot 15\end{array}$ & $\begin{array}{r}2 r \cdot 8 \dagger \\
\pm I \cdot 19\end{array}$ & $\begin{array}{c}24 \cdot 2 \\
\pm 1 \cdot 05\end{array}$ & $\begin{array}{l}25 \cdot 0 \\
\pm 0.45\end{array}$ \\
\hline $40 \mathrm{mg}$ & $\begin{array}{c}30.5 \\
\pm 0.72\end{array}$ & $\begin{array}{l}28 \cdot 8 \\
\pm 0.79\end{array}$ & $\begin{array}{l}2 r \cdot 0 \dagger \\
\pm 1 \cdot 00\end{array}$ & $\begin{array}{r}20 \cdot 3 \ddagger \\
\pm 1 \cdot 02\end{array}$ & $\begin{array}{r}19 \cdot 0 \ddagger \\
\pm 0 \cdot 77\end{array}$ & $\begin{array}{r}20 \cdot 0^{*} \\
\pm \mathrm{r} .55\end{array}$ & $\begin{array}{r}22 \cdot 8^{*} \\
\pm \mathrm{r} \cdot 25\end{array}$ \\
\hline $80 \mathrm{mg}$ & $\begin{array}{l}29.7 \\
\pm 0.84\end{array}$ & $\begin{array}{l}29 \cdot 7 \\
\pm 1 \cdot 41\end{array}$ & $\begin{array}{l}22 \cdot 7 \\
\pm 1 \cdot 74\end{array}$ & $\begin{array}{r}18 \cdot 5 \ddagger \\
\pm 1 \cdot 06\end{array}$ & $\begin{array}{r}17 \cdot 2 \ddagger \\
\pm 1 \cdot 14\end{array}$ & $\begin{array}{r}18.7 \pm \\
\pm 1.05\end{array}$ & $\begin{array}{r}19.5 \ddagger \\
\pm 0.85\end{array}$ \\
\hline
\end{tabular}

Values expressed as mean $\pm \mathrm{SEM}(\mathrm{mmHg})$. Statistical significance expressed as * $(P<0.05) ; \uparrow(P<0.01) ; \ddagger(P<0.001)$

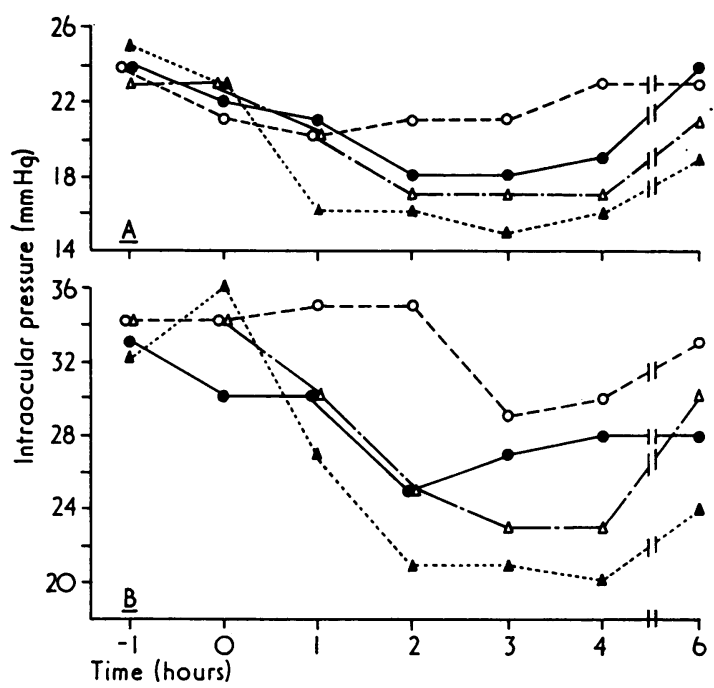

FIG. I The time course of the IOP response in two cases, one from each pressure group, recorded from the eye with the highest IOP after treatment with placebo and different doses of propranolol. The substances were administered at $T=0$ hour

Placebo ( $\mathrm{O}-\mathrm{O}$ ), $20 \mathrm{mg}$ propranolol ( $\mathrm{O}-\mathrm{O}$ ), $40 \mathrm{mg}$ propranolol $(\triangle \longrightarrow \triangle)$, $80 \mathrm{mg}$ propranolol ( $\triangle-\Delta)$.

(A) Initial highest IOP ranging 20 to $29 \mathrm{mmHg}$.

(B) Initial highest IOP ranging 30 to $39 \mathrm{mmHg}$

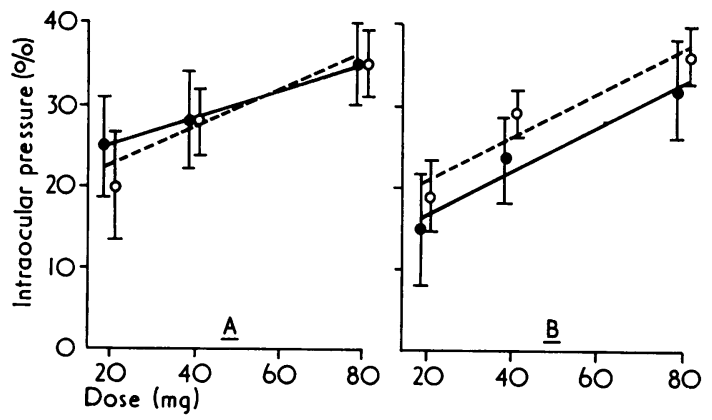

FIG. 2 Mean maximum IOP decrease $\pm S E M$ in per cent after administration of different doses of propranolol

Initial highest IOP ranging 20 to $29 \mathrm{mmHg}\left(\mathrm{O}_{-}\right)$). Initial highest IOP ranging 30 to $39 \mathrm{mmHg}\left(\mathrm{O}_{-}-\mathrm{C}_{\mathrm{O}}\right)$. (A) Eye with the highest IOP. (B) Fellow eye

reached its maximum after two hours. The fall in pulse rate showed a clear correlation with the doses given (Table IV).

Differences were statistically significant at doses of 40 and $80 \mathrm{mg}$.

\section{Discussion}

The results of the present study indicate that orally-administered propranolol causes a statistically 
Table III The time-course of the systolic blood pressure response of 12 ocular hypertensive patients after treatment with placebo and different doses of propranolol

\begin{tabular}{|c|c|c|c|c|c|}
\hline \multirow{2}{*}{$\begin{array}{l}\text { Initial } \\
\text { highest } \\
I O P(\mathbf{m m H g})\end{array}$} & \multirow{2}{*}{ Dosage } & \multicolumn{4}{|l|}{ Hours } \\
\hline & & 0 & $\mathbf{I}$ & 3 & 6 \\
\hline \multirow[t]{4}{*}{$20-29$} & Placebo & $\begin{array}{l}171 \\
\pm 5.23\end{array}$ & $\begin{array}{l}166 \\
\pm 5.39\end{array}$ & $\begin{array}{l}168 \\
\pm 6 \cdot 29\end{array}$ & $\begin{array}{l}167 \\
\pm 6.01\end{array}$ \\
\hline & $20 \mathrm{mg}$ & $\begin{array}{l}161 \\
\pm 3 \cdot 27\end{array}$ & $\begin{array}{l}155 \\
\pm 3.42\end{array}$ & $\begin{array}{l}158 \\
\pm 5 \cdot 59\end{array}$ & $\begin{array}{l}161 \\
\pm 6.38\end{array}$ \\
\hline & $40 \mathrm{mg}$ & $\begin{array}{l}155 \\
\pm 5.48\end{array}$ & $\begin{array}{l}153 \\
\pm 4.41\end{array}$ & $\begin{array}{l}143 \dagger \\
\pm 3.59\end{array}$ & $\begin{array}{l}159 \\
\pm 6.88\end{array}$ \\
\hline & $80 \mathrm{mg}$ & $\begin{array}{l}159 \\
\pm 8.60\end{array}$ & $\begin{array}{l}160 \\
\pm 5 \cdot 16\end{array}$ & $\begin{array}{l}153 \\
\pm 6.29\end{array}$ & $\begin{array}{l}156 \\
\pm 8 \cdot 70\end{array}$ \\
\hline \multirow[t]{4}{*}{$30-39$} & Placebo & $\begin{array}{l}158 \\
\pm 6.67\end{array}$ & $\begin{array}{l}158 \\
\pm 8.63\end{array}$ & $\begin{array}{l}153 \\
\pm 6 \cdot 16\end{array}$ & $\begin{array}{l}149 \\
\pm 6.76\end{array}$ \\
\hline & $20 \mathrm{mg}$ & $\begin{array}{l}151 \\
\pm 8.41\end{array}$ & $\begin{array}{l}150 \\
\pm 8.56\end{array}$ & $\begin{array}{l}140 \\
\pm 7.53\end{array}$ & $\begin{array}{l}144 \\
\pm 8.60\end{array}$ \\
\hline & $40 \mathrm{mg}$ & $\begin{array}{l}152 \\
\pm 9.37\end{array}$ & $\begin{array}{l}142 \\
\pm 8.63\end{array}$ & $\begin{array}{l}138 \\
\pm 9.01\end{array}$ & $\begin{array}{l}151 \\
\pm 9.08\end{array}$ \\
\hline & $80 \mathrm{mg}$ & $\begin{array}{l}156 \\
\pm 6.88\end{array}$ & $\begin{array}{l}149 \\
\pm 7 \cdot 24\end{array}$ & $\begin{array}{l}145 \\
\pm 7.53\end{array}$ & $\begin{array}{c}148 \\
\pm 11 \cdot 24\end{array}$ \\
\hline
\end{tabular}

Values expressed as mean $\pm \mathrm{SEM}(\mathrm{mmHg})$. Statistical significance expressed as $+(P<0.01)$

Table IV The time-course of the decrease in pulse rate of 12 ocular hypertensive patients after treatment with placebo and different doses of propranolol

\begin{tabular}{|c|c|c|c|c|c|c|}
\hline \multirow{2}{*}{$\begin{array}{l}\text { Initial } \\
\text { highest } \\
\text { IOP }(m m H g)\end{array}$} & \multirow{2}{*}{ Dosage } & \multicolumn{5}{|l|}{ Hours } \\
\hline & & 0 & I & 2 & 4 & 6 \\
\hline \multirow[t]{4}{*}{$20-29$} & Placebo & $\begin{array}{c}68 \cdot 3 \\
\pm 4 \cdot 57\end{array}$ & $\begin{array}{c}68.7 \\
\pm 4.70\end{array}$ & $\begin{array}{c}67 \cdot 7 \\
\pm 4 \cdot 18\end{array}$ & $\begin{array}{c}68 \cdot 7 \\
\pm 4.86\end{array}$ & $\begin{array}{c}71 \cdot 3 \\
\pm 3.99\end{array}$ \\
\hline & $20 \mathrm{mg}$ & $\begin{array}{c}62 \cdot 3 \\
\pm 2 \cdot 60\end{array}$ & $\begin{array}{c}58.7 \\
\pm 3.45\end{array}$ & $\begin{array}{c}59.0 \\
\pm 3.42\end{array}$ & $\begin{array}{c}59.3 \\
\pm 3.53\end{array}$ & $\begin{array}{c}6 r \cdot 3 \\
\pm 3 \cdot 25\end{array}$ \\
\hline & $40 \mathrm{mg}$ & $\begin{array}{c}61 \cdot 7 \\
\pm 2 \cdot 85\end{array}$ & $\begin{array}{c}57 \cdot 0 \\
\pm 3.49\end{array}$ & $\begin{array}{r}55 \cdot 0^{*} \\
\pm 3.00\end{array}$ & $\begin{array}{c}60.3 \\
\pm 3.07\end{array}$ & $\begin{array}{c}62 \cdot 7 \\
\pm 3 \cdot 37\end{array}$ \\
\hline & $80 \mathrm{mg}$ & $\begin{array}{c}63.0 \\
\pm 3.53 \\
\end{array}$ & $\begin{array}{r}55.7^{*} \\
\pm 2.99\end{array}$ & $\begin{array}{r}53.3^{*} \\
\pm 2.81\end{array}$ & $\begin{array}{l}58 \cdot 3 \\
\pm 4.05\end{array}$ & $\begin{array}{c}6 r \cdot 3 \\
\pm 3 \cdot 64 \\
\end{array}$ \\
\hline \multirow[t]{4}{*}{$30-39$} & Placebo & $\begin{array}{c}80.0 \\
\pm 5.24\end{array}$ & $\begin{array}{c}79.0 \\
\pm 5.63\end{array}$ & $\begin{array}{c}74.7 \\
\pm 5.43\end{array}$ & $\begin{array}{c}78.7 \\
\pm 4.34\end{array}$ & $\begin{array}{c}80.0 \\
\pm 3.76\end{array}$ \\
\hline & $20 \mathrm{mg}$ & $\begin{array}{c}70 \cdot 3 \\
\pm 3 \cdot 28\end{array}$ & $\begin{array}{c}67 \cdot 0 \\
\pm 3 \cdot 68\end{array}$ & $\begin{array}{c}63.3 \\
\pm 1.91\end{array}$ & $\begin{array}{c}69.0 \\
\pm 4.09\end{array}$ & $\begin{array}{c}72 \cdot 0 \\
\pm 3 \cdot 22\end{array}$ \\
\hline & $40 \mathrm{mg}$ & $\begin{array}{c}71 \cdot 7 \\
\pm 5.67\end{array}$ & $\begin{array}{c}65 \cdot 0 \\
\pm 4.19\end{array}$ & $\begin{array}{r}59.3^{*} \\
\pm 4.22\end{array}$ & $\begin{array}{c}65.7 \\
\pm 3.91\end{array}$ & $\begin{array}{c}69.0 \\
\pm 4.49\end{array}$ \\
\hline & $80 \mathrm{mg}$ & $\begin{array}{l}73.3 \\
\pm 5.10\end{array}$ & $\begin{array}{r}59.3 \dagger \\
\pm 0.84\end{array}$ & $\begin{array}{r}59.3^{*} \\
\pm 2.56\end{array}$ & $\begin{array}{r}64.0^{\circ} \\
\pm 3.54\end{array}$ & $\begin{array}{r}66.0^{*} \\
\pm 4.23\end{array}$ \\
\hline
\end{tabular}

Values expressed as mean \pm SEM (beats/min). Statistical significance expressed as * $(P<0.05) ; t(P<0.01)$

significant decrease of IOP in intraocular hypertensive patients which is clearly dose-dependent. The reduction was appreciable one hour after administration and reached its maximum after three hours at all dose levels tested. It was still present six hours after the administration. This is in agreement with the recently published study by Cuthbert and Collins (1975) in which the plasma level after a single oral dose of propranolol $(40 \mathrm{mg}$ ) was at its highest after two to three hours. Also the maximum degree of beta-adrenoreceptor blockade as judged from the effect on the pulse rate (isoprenaline ratio) was observed at approximately the same time. However, orally-administered propranolol undergoes hepatic metabolism with conversion of drug to active metabolites (Fitzgerald and O'Donnel, 1971) and, therefore, the drug plasma level may not reflect the total beta-inhibitory effect of the substance. Topically applied propranolol is able to bring about a decrease in IOP which is noticeable after one hour and has maximum effect after two to three hours (Vale and others, 1972). Similar time course of IOP response after intravenous administration in man was reported by Vale and Phillips (1970) although no obvious doserelationship could be demonstrated in these experiments; this discrepancy was explained by the fact that two different doses ( $\mathrm{I}$ and $4 \mathrm{mg}$ ) of propranolol were used and administered at short intervals so when the second and largest dose was given the intraocular pressure was already reduced and, therefore, a further decrease proportional to this dose was difficult to achieve.

In agreement with Coté and Drance (1968) we found an evident reduction of IOP after even the smallest dose $(20 \mathrm{mg})$ in both the groups. Noteworthy was the fact that the absolute mean IOP fall during treatment with similar doses of propranolol was greater in the group with higher initial IOP, a finding also reported by Coté and Drance (1968).

The decrease in systolic and diastolic blood pressure was only moderate and never attained statistical significance with one exception. Nor was any dose-relationship demonstrated.

The simultaneous recording of heart rate in the supine position showed during treatment a decrease which was significant in both the groups for the doses of 40 and $80 \mathrm{mg}$. The mean maximum fall was shown to be after one to two hours with an obvious dose-dependence.

The virtual absence of a significant decrease in blood pressure and the lack of correlation with the doses administered suggests that the fall in blood pressure is not the primary cause of the reduced IOP.

\section{Summary}

Placebo and propranolol (Inderal $\otimes$ ) in doses of 20 , 40 , and $80 \mathrm{mg}$ were given in a single-blind test to two groups of six ocular hypertensives. The groups 
consisted of patients with an intraocular pressure ranging from 20 to $29 \mathrm{mmHg}$ and 30 to $39 \mathrm{mmHg}$. The doses were given 48 hours apart and administered after fasting 12 hours. IOP by Goldmann applanation tonometer, systemic blood pressure and pulse rate in the supine position were recorded hourly before and after administration. In both groups a decrease in mean IOP was noted after one hour and this reduction reached its i

\section{References}

BIETTI, G. (1972) Amer. F. Ophthal., 73, 475

COTE, G., and DRANCE, S. (1968) Canad. Y. Ophthal., 3, 207

CUTHBERT, M. F., and collins, R. F. (1975) Brit. F. clin. Pharmacol., 2, 49

FitzGRRALD, J. D., and o'DONNEL, S. (1971) Brit. F. Pharmacol., 43, 222

MUSINI, A., FABBRI, B., BERGAMASCHI, M., MANDELLI, v., and SHANKS, R. G. (1971) Amer. F. Ophthal., 72, 773

ÖHRSTRÖM, A. (1973) Acta ophthal. (Kbh.), 51, 639

PANDOLFI, M., and ÖHRSTRÖM, A. (1974) Ibid., 52, 464

PHILliPS, C. I., HOWITT, G., and ROWLANDS, D. J. (1967) Brit. F. Ophthal., 51, 222

SHARAF, E., HAROUN, E., ISHAAC, z., EL SHEWY, T., and NASSEF, A. (1974) Exp. Eye Res., 19, 223

VALE, J., GIBBS, A. C. C., and PHILlIPS, C. I. (1972) Brit. Y. Ophthal., 56, 770 , and PHILlips, c. 1. (1970) Exp. Eye Res., 9, 82

WETTRELL, K., and PANDOLFI, M. (1975) Ibid., 2I, 45 I 